\title{
Method of Termination of Life
}

National Cancer Institute

\section{Source}

National Cancer Institute. Method of Termination of Life. NCI Thesaurus. Code C90423.

The mechanism by which a life is ended. 